\title{
Quantum entanglement and a metaphysics of relations
}

\author{
Michael Esfeld \\ University of Lausanne \\ Department of Philosophy \\ CH-1015 Lausanne, Switzerland \\ Michael-Andreas.Esfeld@philo.unil.ch
}

(forthcoming in Studies in the History and Philosophy of Modern Physics 35B (Dec. 2004))

\begin{abstract}
This paper argues for a metaphysics of relations based on a characterization of quantum entanglement in terms of non-separability, thereby regarding entanglement as a sort of holism. By contrast to a radical metaphysics of relations, the position set out in this paper recognizes things that stand in the relations, but claims that, as far as the relations are concerned, there is no need for these things to have qualitative intrinsic properties underlying the relations. This position thus opposes a metaphysics of individual things that are characterized by intrinsic properties. A principal problem of the latter position is that it seems that we cannot gain any knowledge of these properties insofar as they are intrinsic. Against this background, the rationale behind a metaphysics of relations is to avoid a gap between epistemology and metaphysics.

Keywords: entanglement, holism, intrinsic properties, metaphysics, non-separability, relations, structural realism
\end{abstract}

\section{Introduction}

The aim of this paper is to propose a philosophical characterization of quantum entanglement within a broader metaphysical framework. The framework is the one of a metaphysics of relations in contrast to a metaphysics of individual things that are characterized by intrinsic properties. A metaphysics of relations is a minority view in contemporary philosophy. According to the mainstream of metaphysical thought, the world consists of independent individual things that are embedded in space-time. These things are individuals, because (a) they have a spatio-temporal location, (b) they are a subject of the predication of properties each and (c) there are some qualitative properties by means of which each of these things is distinguished from all the other ones (at least the spatio-temporal location is such a property).

Qualitative properties are all and only those properties whose instantiation does not depend on the existence of any particular individual; properties such as being that individual are hence excluded. These things are independent, because their basic properties are intrinsic ones. Basic properties are fundamental in that they are not reducible to other properties, and they are not disjunctive; that is to say, properties such as "being round or square" are excluded. Intrinsic are all and only those qualitative properties that a thing has irrespective of whether or not there are other contingent things; all other qualitative properties are extrinsic or relational. That is to say: Having or lacking an intrinsic property is independent of accompaniment or loneliness. ${ }^{1}$

1 See Langton and Lewis (1998) and for a refinement Lewis (2001). 
A metaphysics of this type can be traced back to Aristotle at least. Aristotle assumes that there is a plurality of individual things (substances) that are characterized by intrinsic properties (forms) each. ${ }^{2}$ A prominent contemporary conception is David Lewis' thesis of Humean supervenience: ${ }^{3}$ at the basic level of the world, there are only local qualities in the sense of intrinsic properties instantiated by space-time points or point-sized particles or field sources at space-time points. Space-time points can qualify as individual things in the abovementioned sense. Everything there is in a world like ours supervenes on the distribution of basic intrinsic properties over all space-time points. Whether really everything supervenes on that distribution is not relevant to the present paper. What is important here is the claim that, except for spatio-temporal relations, all the relations between the things at the basic level supervene on their intrinsic properties.

It is well known that quantum physics - notably quantum entanglement - poses a challenge to Lewis' Humean supervenience. This paper argues that quantum physics can be taken to suggest replacing a metaphysics of intrinsic properties with a metaphysics of relations. This is, of course, a matter of weighing arguments in the interpretation of a physical theory. There is no direct route of logical implication from physics to a particular metaphysics. A metaphysics of relations is often dismissed out of hand, for it seems to be paradoxical. It seems that (a) relations require relata, that is, things which stand in the relations, and that (b) these things have to be something in themselves, that is, must have intrinsic properties over and above the relations in which they stand. ${ }^{4}$ However, a metaphysics of relations merely has to reject the second part of this claim: one can maintain that (a) relations require relata, that is, things which stand in the relations, but that $\left(b^{*}\right)$ these things do not have any intrinsic properties that underlie the relations in which they stand. There is nothing paradoxical in this latter claim. This paper argues for no more than this claim.

By a "thing", I mean anything that is a subject of the predication of properties, including relational properties (relations), without being itself predicated as a property of something. I do not distinguish relations from relational properties: relations are properties as well (albeit not monadic ones) in that they are predicated of things. Moreover, something can be a thing without being an individual thing; for something to be an individual thing (an individual), further conditions may have to be met such as (a) being distinguishable from all the other things by means of the predication of some qualitative properties or (b) having a primitive thisness (haecceity). ${ }^{5}$

A metaphysics of relations can grant that things may have non-qualitative properties over and above the relational ones such as the property of being this thing, that is, a primitive thisness; but this position does not have to admit primitive thisness. Accepting that relations require things which stand in the relations does not commit one to the view that these things have a primitive thisness. It simply means taking into account that properties, including relations, are predicated of something; this does not imply that there is more to the related things than standing in the relations. If one does not endorse primitive thisness, one may say that a thing is a bundle of properties (or tropes); how a thing can be a bundle of relational properties (or relational tropes) is no more - and no less - a problem than how it can be a

2 See in particular Categories, chapter 5, and Metaphysics, book VII.

3 See Lewis (1986a), pp. IX-X.

4 See, for instance, Langton (1998).

5 See Adams (1979). 
bundle of intrinsic properties (or intrinsic tropes). Hence, both the view of things as being characterized by primitive thisness and the view of things as bundles of properties (or tropes) are compatible with a metaphysics of relations.

The plan of the paper is as follows: Starting from a conceptualization of quantum entanglement in terms of non-supervenient relations, the next section argues for conceiving quantum entanglement as a case of non-separability (that is, a case of holism). It is shown how this interpretation of quantum entanglement leads to a metaphysics of relations. The third section then establishes that this is a moderate metaphysics of relations in contrast to a radical one. Finally, this interpretation of quantum entanglement is put into the broader framework set out in this introduction: it is argued that this interpretation avoids a gap between epistemology and metaphysics, whereas a metaphysics of intrinsic properties invites such a gap.

\section{From quantum entanglement to relations without underlying intrinsic properties}

\subsection{Quantum entanglement}

Quantum theory permits that the states of quantum systems are entangled. Instead of speaking of entangled states, one can also talk directly in terms of entangled systems. However, since entanglement is state-dependent, it seems more appropriate to use the notion of entangled states. Entanglement is to say that the quantum systems in question do not have some statedependent properties each independently of one another. Examples are position and momentum as well as spin angular momentum in any direction. Instead, there are only correlations between the state-dependent properties of the quantum systems in question encoded in a joint probability distribution determined by the joint state. Quantum theory does not include any properties of each quantum system taken separately that are a supervenience basis for these correlated probability distributions. Furthermore, these correlations - and thus entanglement - are independent of spatio-temporal distance. Because of entanglement, quantum physics seems to exhibit some sort of a holism.

This way of receiving quantum theory commits us to realism: there are quantum systems, and they are as quantum theory describes them, namely subject to entanglement. Furthermore, we are committed to endorsing objective probabilities, that is, probabilities which do not simply indicate limits of our knowledge, but which are about properties that things objectively have. Whatever entanglement may exactly be, it is a relation among quantum systems. "Being entangled with" is a property that is predicated of at least two quantum systems; it is thus a relational property. By admitting entanglement, we are not committed to taking a particular stance on the notorious measurement problem in quantum theory: even if one maintains that measurement leads to a dissolution of entanglement so that, as a result of measurement, quantum systems really have definite numerical values of the state-dependent properties in question each, entanglement has to be there in the first place before it makes sense to consider the question whether or not there are processes that dissolve entanglement.

It is not necessary that the states of quantum systems are entangled. Quantum theory has the means at its disposal to describe states of physical systems which are not entangled, that is, product states. However, quantum theory describes physical systems in such a way that entanglement is not at all exceptional, but ubiquitous. What has to be accounted for in quantum theory is not entanglement, but cases of the absence of entanglement, if there really are such cases (if not, it has to be explained why there appear to be such cases). 
Nonetheless, even if the states of quantum systems are entangled, it is possible to give a description of each of the systems in question considered in isolation from the other systems. This is a description in terms of what is known as a mixed state in the sense of an improper mixture: 6 this description contains all the information that can be acquired about each of the quantum systems considered on its own. But it ignores the correlations in which the entanglement consists. Consequently, this description does not take all the factors into account that are relevant to the quantum probabilities. The description in terms of improper mixtures therefore is an incomplete description of quantum systems in entangled states. It is not a description that refers to intrinsic properties of each quantum system.

\section{$2.2 \quad$ Non-supervenient relations}

A prominent philosophical proposal for a characterization of quantum entanglement is the one of Paul Teller (1986). He describes quantum entanglement in terms of relational holism, taking it to consist in non-supervenient relations:

Holism has always seemed incoherent, for it seems to say that two distinct things can somehow be entangled or intermeshed so that they are not two distinct things after all. ... By relational holism I will mean the claim that objects which in at least some circumstances we can identify as separate individuals have inherent relations, that is, relations which do not supervene on the non-relational properties of the distinct individuals. Relational holism is free of the incoherence which threatens less clearly stated forms of holism. It is sufficient for an object to be a distinct individual that it have a non-relational property. And it is quite consistent to suppose that two such distinct individuals, each having a non-relational property, should also stand in some inherent relation to each other. (p. 73)

Teller thus regards quantum systems as distinct individuals. He claims that what is peculiar about these individuals is that they bear some non-supervenient relations to each other. Hence, according to Teller, it is necessary that quantum systems have some intrinsic properties or other, which make them distinct individuals, in order to be able to stand in the relations of quantum entanglement, even if these relations do not supervene on the intrinsic properties. He suggests that if the presupposition of intrinsic properties that make quantum systems distinct individuals were abandoned, the claim of holism in quantum physics would be an incoherent position.

Consider furthermore what David Mermin (1998) proposes as the Ithaca interpretation of quantum mechanics, namely that quantum theory describes a world of correlations without describing intrinsic properties of the correlata. ${ }^{7}$ Mermin goes on to say that "the correlata that underlie those correlations lie beyond the descriptive powers of physical science" (p. 762) and that "in our description of nature the purpose is not to disclose the real essence of the phenomena" (p. 764). Again, the idea is that quantum theory describes relations and that these relations presuppose intrinsic properties of the related systems, which are beyond the scope of quantum theory.

However, the characterization as a non-supervenient relation is not sufficient to capture quantum entanglement. Spatio-temporal relations, for instance, are non-supervenient, too. Independently of quantum theory, Carol Cleland (1984) introduces a distinction between weakly and strongly non-supervenient relations. She employs the familiar philosophical

$7 \quad$ See also Rovelli (1996). 
differentiation between a determinable attribute - such as having mass - and a determinate attribute - such as having a mass of $6 \mathrm{~g}$ : nothing has mass simpliciter, but only in a determinate way. She formalizes the concept of a supervenient relation by setting out two conditions:

A dyadic relation $R$ is supervenient upon a determinable nonrelational attribute $P$ if and only if

1. $\square(\square x, y) \square \diamond\left[R(x, y)\right.$ and there are no determinate attributes $P_{i}$ and $P_{j}$ of determinable kind $P$ such that $P_{i}(x)$ and $P_{j}(y)$ ];

2. $\square(\square x, y)\left\{R(x, y) \quad\right.$ there are determinate attributes $P_{i}$ and $P_{j}$ of determinable kind $P$ such that $P_{i}(x)$ and $P_{j}(y)$ and $\square(\square x, y)\left[\left(P_{i}(x)\right.\right.$ and $\left.\left.\left.P_{j}(y)\right) \quad R(x, y)\right]\right\}$. (p. 25)

The first condition states: it is necessary for a supervenient relation that each of the related things instantiates a determinate non-relational - that is, intrinsic - property of a certain determinable kind. The second condition adds that these instantiations of intrinsic properties determine the relation in question. It is necessary that any two things which instantiate the same intrinsic properties stand in the same relation to each other. Cleland gives the example of mass relations. An individual that has a mass of $8 \mathrm{~g}$ is heavier than an individual that has a mass of $6 \mathrm{~g}$. Any two individuals that have the same masses respectively instantiate the same mass relation. Therefore, mass relations are supervenient on the property of mass, which can be considered as intrinsic for the purpose of the argument (pp. 23-27).

Coming to non-supervenience, a relation is weakly non-supervenient if and only if it satisfies the first, but not the second of these conditions. That is to say: it is necessary that all the related things instantiate intrinsic properties of a certain kind. But these intrinsic properties are not sufficient to determine the relation in question. Cleland gives the following example: according to her, if two individuals are to bear a relation of spatial distance to each other, each of them must have a size or shape. However, their size or shape does not determine the spatial distance between them. Therefore, Cleland claims that the relation of spatial distance is weakly non-supervenient: this relation satisfies the first condition for a supervenient relation, but it fails to conform to the second one (pp. 27-28).

Accordingly, a relation is strongly non-supervenient if and only if it does not satisfy the first condition for a supervenient relation either (pp. 28-29). It is thus not necessary that two things have intrinsic properties of a certain kind, if they bear a strongly non-supervenient relation to each other. Strongly non-supervenient relations permit that the related things have intrinsic properties each, but they do not require intrinsic properties of a certain kind. Steven French convincingly argues that the non-supervenient relations in the case of quantum entanglement are strongly non-supervenient in Cleland's sense. ${ }^{8}$ Quantum entanglement is not tied to the related systems having intrinsic properties of a certain kind.

Cleland's distinction between weakly and strongly non-supervenient relations may be helpful to differentiate quantum entanglement from spatio-temporal relations. According to Cleland, spatial relations are weakly non-supervenient. The relations of quantum entanglement, by contrast, are strongly non-supervenient. However, this distinction is not sufficient to conceptualize quantum entanglement.

In On the plurality of worlds, David Lewis (1986b) considers whether there can be external relations which are not strictly or analogically spatio-temporal and which unify a world. $\mathrm{He}$ imagines the following test case: 
We tend to think that positive and negative charge are natural intrinsic properties of particles; but suppose not. Suppose instead there are natural external relations of like-chargedness and opposite-chargedness. ... On this view, as opposed to the standard view, the relations of likeand opposite-chargedness do not supervene on the intrinsic natures of two particles taken separately; an electron and a positron may be perfect intrinsic duplicates. That is the point of calling the relations external. They are natural ex hypothesi. They are pervasive (at least, given the appropriate laws) in that whenever two particles are connected by a chain of such relations, they are connected directly. But they are very far from discriminating (again, given the appropriate laws): if there are as few as three particles, there must be two of them that are alike as far as these relations are concerned. If this story, or something like it, could be true, then here we have external relations that are not strictly or analogically spatio-temporal. (p. 77)

Lewis' imaginary charge relations are strongly non-supervenient in Cleland's sense: standing in these relations is not tied to instantiating intrinsic properties of a certain kind. Lewis argues that these imaginary charge relations are unable to unify a world, because there is no convincing reason against considering two things in different possible worlds as also standing in relations of like- or opposite-chargedness to each other (pp. 76-78). In the case of the quantum relations of entanglement, by contrast, there is, like in the case of spatio-temporal relations, no question of these relations holding also among things across different worlds. However, spatio-temporal relations as well as Lewis' imaginary charge relations are stateindependent in the following sense: any two things in a world bear some spatio-temporal relation to each other whatever their state is. By contrast, it is state-dependent whether two things bear a relation of entanglement to each other. Nonetheless, since entanglement is ubiquitous at the microphysical level in all the worlds to which quantum theory applies, we can say: not only spatio-temporal relations, but also quantum relations of entanglement unify our world. Both are non-supervenient relations that connect only things in one world.

Lewis' imaginary charge relations come closer to quantum entanglement than spatiotemporal relations in the following respect: in the case of these charge relations as well as in the quantum case we expect the relations to be determined by properties which each of the related systems has independently of the other systems. In other words, we have a prior understanding of properties such as charge as well as position and momentum as being intrinsic properties. But it then turns out that these properties are not intrinsic and that there are no intrinsic properties which accomplish such a determination. Nonetheless, there is nothing like entanglement in Lewis' example. Lewis simply invites us to imagine a case in which the properties of being like-charged or being opposite-charged with respect to certain other things are irreducibly relational. Hence, Lewis' imaginary charge relations show the following: even if we make an exception for spatio-temporal relations, speaking of nonsupervenient relations is not sufficient to capture what is peculiar about quantum entanglement.

\section{$2.3 \quad$ Non-separability}

The sense in which there is a failure of supervenience in quantum entanglement can be elaborated on in another way than simply speaking of non-supervenient relations. In any case of quantum entanglement, there are no states of the parts on which the joint state of the whole of the systems in question supervenes. We can make this notion of a failure of supervenience more restrictive by employing the concept of separability. 
Albert Einstein based his criticism of quantum theory on the principle of separability, which he regarded as some sort of a presupposition without which physical science would not be possible at all. 9 Taking Einstein's criticism into account, Don Howard formulates separability as the claim that (1) spatially separated systems possess their own, distinct physical state each and that (2) the joint state of two or more spatially separated systems is wholly determined by their separate states. ${ }^{10}$ In view of employing the notion of separability for a systematic characterization of quantum entanglement, it seems reasonable to eliminate the condition of the systems being spatially separated; for entanglement is independent of whether or not the systems whose states are entangled are spatially separated. For instance, the spin state of the two electrons of a helium atom in the groundstate is a case of entanglement, too (singlet state), although the two electrons are not localized in such a way that they are spatially separated from one another. Furthermore, in quantum computation, one considers the entanglement of the states of many systems which are usually not localized in such a way that they are separated in space. Abandoning the condition of spatial separation, one can characterize separability in this way: Physical systems have a state each in the sense that (1) this state completely determines the state-dependent properties of the system and (2) the joint state of two or more systems supervenes on the states which each of these systems has. On this basis, let us characterize non-separability in the following way:

\section{Non-separability}

The states of two or more systems are non-separable if and only if it is only the joint state of the whole that completely determines the state-dependent properties of each system and the correlations among these systems (to the extent that these are determined at all).

According to this characterization, any case of quantum entanglement is a case of nonseparability, and non-separability is the reason why quantum entanglement is a sort of holism. ${ }^{11}$ In any case of quantum entanglement, only the joint state of the whole completely determines the probability distributions of the state-dependent properties of the parts by determining correlations among these probability distributions.

Coming back to Lewis's example of imaginary charge relations mentioned in the previous section, this is simply the case of one system being in a state of, say, opposite-chargedness relative to another system (or other systems). This is comparable to the case when, as a result of measurement and a dissolution of entanglement, there is, in the paradigmatic example of the singlet spin state of a pair of systems of spin 1/2, the one system in a state of opposite spin relative to the other system. However, when the spin states of the two systems are entangled, it is not the case that each system is in a spin state opposite to the other system.

Consider another example: Max Black conceives a possible world in which there are only two spheres that have the same non-relational properties. ${ }^{12}$ There is a definite distance between these two spheres. But neither sphere has a position in distinction from the other sphere (unless one presupposes absolute space). In Black's example, each sphere is in a state of (or has the property of) being at a distance of, say, ten metres from another sphere. In the

9 See in particular Einstein (1948), pp. 321-322, translated in Howard (1985), pp. 187-188.

10 Howard (1989), pp. 225-227.

11 For an elaboration on the link between non-separability and holism, see Esfeld (2001), chapter 8.

12 Black (1952), p. 156. 
example of the states of two quantum systems entangled with respect to position and momentum that Einstein, Podolsky and Rosen (1935) consider in their famous paper, by contrast, even if the global observable of relative distance has the definite numerical value of, say, ten metres, it is not permissible to attribute to each of the two systems the state - and thereby the property - of being at a distance of ten metres from the other system. Imagine a simplified experimental realization of this case. If we attributed to the one system the property of being ten metres apart from the other system and attributed to that other system the property of being ten metres apart from the first system, we would imply that one system is on the one side of the experimental arrangement and the other system on the other side. However, we can ask about the local observable of position of each of the two systems, and we get a probability distribution which is identical for the two systems and which extends to both sides of the experimental arrangement. Hence, in the case of Einstein, Podolsky and Rosen, there is no warrant in the formalism of quantum theory for assuming that the one system is on one side of the arrangement and the other system on the other side of the arrangement. ${ }^{13}$ The cases of quantum entanglement are cases of non-separability in that there is only a joint state determining certain correlations that obtain among the systems in question without it being possible to attribute significant states (or state-dependent properties for that matter) to each of the systems in question, and be it relative to the other systems.

Nonetheless, characterizing what quantum theory tells us in terms of non-separability is a metaphysical proposal that is a matter of philosophical argument. As with any philosophical argument, the experimental evidence - in particular, the analysis of Bell's theorem and the evidence coming from the Bell experiments - does not force a commitment to nonseparability upon us. ${ }^{14}$ My claim is that speaking of non-separability provides us with a convincing understanding of what is going on in quantum entanglement.

One can conceive the joint state of a quantum whole as including intrinsic properties of the whole, that is, intrinsic properties which do not supervene on intrinsic properties of the parts. ${ }^{15}$ These intrinsic properties of the whole, however, do not speak in favour of a metaphysics of intrinsic properties in contrast to a metaphysics of relations: Any relation between two or more systems can be conceived as an intrinsic property of the whole of the systems in question. In quantum entanglement, the intrinsic properties of the whole are global observables such as, in the case of Einstein, Podolsky and Rosen, relative distance and total momentum as well as total spin in the case of the singlet spin state of a pair of systems of spin $1 / 2$. These global observables are represented as a certain combination of local observables of the parts in the Hilbert space formalism. They thus contain information about the way in which the parts are related to each other. Moreover, since quantum entanglement is ubiquitous, strictly speaking, only the whole of all quantum systems taken together can be considered as being in a pure state. Intrinsic properties of the world as a whole at the quantum level, however, do not in any way challenge a metaphysics of relations. No argument for a metaphysics of intrinsic properties can be built on such intrinsic properties.

For any system of a certain kind, there is a family of properties that make something a system of the kind in question. State-dependent properties such as position, momentum, and

13 See also the argument in Cartwright (1989), p. 263.

14 For a view against non-separability see the recent paper by Winsberg and Fine (2003). See, however, Müller and Placek (2001) for an argument against Fine's approach.

15

Compare Healey (1991). 
spin in any direction belong to the family of properties that make something a quantum system. Nothing can count as a quantum physical system without having these properties in some sense. If one countenances essential properties, position, momentum, and spin in any direction count among the essential properties of a quantum physical system. In the case of quantum entanglement, however, quantum systems do not have these properties separately. Nonetheless, these systems taken together have properties of the kind position, momentum, or spin - such as, in the paradigmatic examples mentioned above, relative distance and total momentum as well as total spin.

If asked about position, momentum, or spin in a given direction of one of the two systems in the state that Einstein, Podolsky and Rosen consider or in the singlet state, one can therefore say the following: one system is related with another system in such a way that only the two systems taken together have properties of the kind in question - in the sense of a relative distance with a definite numerical value, total momentum zero, or total spin zero. Nonetheless, the property of relative distance of the whole indicates the way in which the parts are related with respect to position (although it is not the case that each of the parts has a position); for the global observable of relative distance contains the correlations between the probability distributions of the local observables in question, in whatever way one may interpret these correlated probability distributions. The property of total momentum of the whole indicates the way in which the parts are related with respect to momentum, although it is not the case that each of the parts has a momentum - and be it one that is simply opposed to the momentum of the other system. Accordingly, the property of total spin of the whole indicates the way in which the parts are related with respect to spin in any direction, although it is not the case that each of the parts has a spin in any direction - and be it one that is simply opposed to the spin of the other system.

A similar consideration applies to any case of entanglement: there are global observables of the whole that have a definite numerical value in the state in question and that can be considered as intrinsic properties of the whole. These properties of the whole indicate the way in which the parts are related with respect to their state-dependent properties, for they contain correlations between the probability distributions of the respective state-dependent properties of the parts, although these state-dependent properties cannot be attributed to each of the parts.

We can thus set out an account of quantum entanglement in this way:

1) Quantum entanglement shows that there are non-supervenient relations among physical systems over and above the spatio-temporal relations (strongly non-supervenient relations in contrast to weakly non-supervenient relations).

2) The non-supervenient relations of entanglement among the parts of a quantum whole amount to the whole having intrinsic properties that do not supervene on intrinsic properties of the parts.

3) These properties of the whole come to non-separability in the following sense: the parts have some of the properties that belong to the family of properties which make something a quantum system not separately, but only in this way: there are properties of the whole which indicate the manner in which the parts are related with each other with respect to some of the properties that make something a quantum system.

If we spell out the non-supervenient relations in the quantum case in terms of non-separability and thus holism as proposed here, quantum theory is no longer neutral with respect to the 
issue of a metaphysics of intrinsic properties vs. a metaphysics of relations. Quantum theory, interpreted in terms of non-separability, speaks in favour of a metaphysics of relations that do not require any intrinsic properties of the related quantum systems. As far as the properties that are subject to entanglement are concerned, there is no reason to suppose that there are intrinsic properties of the related systems in question: the relations among the systems are determined from above so to speak, namely by the pure state of the whole. This way of determining the relations makes it superfluous to call for intrinsic properties of the related systems, even if there is no question of intrinsic properties constituting a supervenience basis for the relations. All we need for these relations to obtain is systems in the sense of entities of which properties can be predicated, and be it relational properties such as "being entangled with". There is no need for the systems of which such properties can be predicated to be distinct individuals.

Quantum systems have more properties than the state-dependent ones, which are subject to entanglement, namely state-independent properties such as mass or charge. One may wonder whether these are intrinsic properties. However that may be, bringing state-independent properties into focus can at most show that quantum systems may have intrinsic properties that are not relevant to the correlations that quantum theory describes. Thus, referring to stateindependent properties can at most illustrate the following point: if one puts forward an argument for a metaphysics of relations on the basis of a physical theory, one cannot exclude that the physical systems in question have some intrinsic property or other. What one can seek to establish is only that the relations which the physical theory in question treats do not call for any intrinsic properties of the related systems. Nonetheless, since quantum theory is our basic physical theory, it would be desirable to derive state-independent properties within the formalism of quantum theory. The idea then is to get to state-independent properties such as charge and mass on the basis of state-dependent properties.

Non-relativistic quantum mechanics can be taken to describe single physical systems such as electrons, neutrons, protons and the like. These are single physical systems, because, as far as quantum mechanics is concerned, there always is a definite number of them. They are subjects of the predication of properties each - and be it properties such as "is entangled with other systems". Quantum systems of the same kind whose states are entangled are indistinguishable. There are no qualitative properties whatsoever - not even relational conditional probabilities - that distinguish one such system from all the other ones. Nonetheless, one can maintain that quantum systems are individuals if one is prepared to acknowledge non-qualitative properties such as primitive thisness. ${ }^{16}$ The proposal made in this section is compatible with such a view. But the point is that it does not commit us to more than acknowledging that, as far as quantum physics is concerned, quantum systems are those things that stand in the correlations without any intrinsic properties or anything like a primitive thisness being required for standing in the correlations. Hence, both the friends of non-individuals and the friends of individuals can agree with the metaphysics of relations proposed in this section. It is important to disentangle the issue of quantum entanglement from the issue of individuals: entanglement rules out that there are individuals that are distinguished by some qualitative properties, but it does not exclude that there may be individuals tout court. 


\section{A moderate vs. a radical metaphysics of relations}

The position argued for in the preceding section comes close to what Steven French and James Ladyman advocate as metaphysical or ontic structural realism, namely the view that structure is what is real and that there are no intrinsic properties underlying structure. ${ }^{17}$ Their argument for this position makes also use of quantum entanglement. Ladyman (1998) concludes by envisaging that "structural realism amounts to the claim that theories tell us not about the objects and properties of which the world is made, but directly about structure and relations" (p. 422), suggesting that there is no need to admit objects in metaphysics. The structural realism of French and Ladyman thus seems to be a radical metaphysics of relations. ${ }^{18}$ It rejects not only the claim that (1) things have to be something in themselves, that is, must have intrinsic properties over and above the relations in which they stand, but also the claim that (2) relations require relata, that is, things which stand in the relations. The metaphysics of relations advocated in the preceding section, by contrast, dismisses only the first claim. It therefore is a moderate metaphysics of relations. The argument of this paper accepts that relations require things that stand in the relations (although these things do not have to be individuals, and they need not have intrinsic properties) and regards physical theories as referring to things. In particular, the argument of the preceding section says nothing against quantum theory referring to quantum systems and describing the properties of these systems, albeit - somewhat peculiar - relational properties. In other words, an argument that builds on quantum entanglement seems to be sufficient only to renounce (1), but does not touch upon (2). At least as far as quantum mechanics is concerned, there are quantum systems that stand in the relations, even if it can be maintained that they fall short of being individuals. (Quantum field theory would in any case require a separate treatment).

French and Ladyman base their ontic structural realism on structural realism as set out by John Worrall (1989). Worrall's structural realism is motivated by two considerations: to take up the 'no miracle argument' for scientific realism, that is the argument that the predictive success of our physical theories would be a miracle if they were not tracking truth; and to pay heed to the 'argument from pessimistic induction', that is the claim that since most of our past physical theories have turned out to be false, it is likely that our present physical theories will endure the same fate. According to Worrall, what is preserved in theory change is structure. Consequently, we should be realists with respect to the structure of our physical theories. This argument for scientific realism hangs upon the structure of a physical theory being distinguished as that what is preserved in theory change from something that is not preserved. In Worrall, the contrast is between structure and nature. He writes, "the structural realist ... insists that it is a mistake to think that we can ever 'understand' the nature of the basic furniture of the universe" (p. 122). This is the old distinction again between structure or relations that can be known and intrinsic properties of the related things that cannot be known (cf. Mermin's Ithaca interpretation of quantum theory mentioned in section 2.2 above). French and Ladyman advance their ontic structural realism in order to overcome that distinction. Their motivation, as well as the motivation of this paper, is to avoid being committed to intrinsic properties of which we cannot gain any knowledge insofar as their intrinsic nature is concerned. Nonetheless, in this context, the point of this paper is that in

See Ladyman (1998), French and Ladyman (2003).

For another contemporary version of a radical metaphysics of relations see Dipert (1997). 
order to get this result, we do not have to jettison the admission of relata, that is, things which stand in the relations.

By way of consequence, however, if we give up Worrall's distinction between structure and nature, the argument for a metaphysics of relations can as such not say anything in defence of scientific realism against the challenge posed by the 'argument from pessimistic induction' apart from making clear that there is no reason to abandon scientific realism as a result of the advent of quantum theory. If quantum theory is superseded by another fundamental physical theory, it may be that the claim that our basic physical theory speaks against intrinsic properties underlying the relations is no longer defensible, because an argument such as the sketched one from quantum entanglement to non-separability would then no longer be available. Any metaphysics that builds on science runs such a risk.

\section{Avoiding a gap between epistemology and metaphysics}

Let us come back to the broader metaphysical framework of a metaphysics of relations vs. a metaphysics of intrinsic properties. Let us assume, in contrast to what has been argued for so far in this paper, that the world at the basic level consists of independent things. How do we gain knowledge of their intrinsic properties? Consider the following problem that Frank Jackson (1998) among others raises:

When physicists tell us about the properties they take to be fundamental, they tell us what these properties $d o$. This is no accident. We know about what things are like essentially through the way they impinge on us and our measuring instruments. It does not follow from this that the fundamental properties of current physics, or of 'completed' physics, are causal cum relational ones. It may be that our terms for the fundamental properties pick out the properties they do via the causal relations the properties enter into, but that at least some of the properties so picked out are intrinsic. They have, as we might put it, relational names but intrinsic essences. However, it does suggest the possibility that (i) there are two quite different intrinsic properties, $P$ and $P^{*}$, which are exactly alike in the causal relations they enter into, (ii) sometimes one is possessed and sometimes the other, and (iii) we mistakenly think that there is just one property because the difference does not make a difference (as the point is put in information theory). An obvious extension of this possibility leads to the uncomfortable idea that we may know next to nothing about the intrinsic nature of the world. We know only its causal cum relational nature. (pp. 2324)

The core of this argument can be reconstructed as follows: (1) We gain empirical knowledge owing to the causal relations that obtain between physical things and our senses. (2) Knowledge thus gained may refer to intrinsic properties of physical things. (3) But the way in which that knowledge is caused imposes a constraint on its content: physical properties can be identified only through the relations in which they enter. If we explain the meaning of the propositions that refer to the fundamental physical properties, it turns out that these propositions describe these properties as relational. (4) Identity of relations, however, does not imply identity of intrinsic properties. (5) We therefore do not know the properties of physical things insofar as they are intrinsic. In other words, we are ignorant of the intrinsic natures of things.

The argument is not that since we gain knowledge through the way in which empirical things impinge on our senses, we know only the way in which they are related to us. The argument is one about the content of empirical predicates, namely that they reveal only 
relations among things. The argument applies to all relations; the relations in which things stand to us do not have any special status as far as the content of empirical knowledge is concerned. To illustrate the claim, one might say that charge, for instance, is the property that makes things attract and repulse one another, mass the property that makes them resist acceleration, etc.

The argument hence contains two claims: a claim about the causes of empirical knowledge and a claim about its content. The causal claim is uncontroversial; the claim about content is more controversial. Let us nonetheless assume that the argument of Jackson (and others), if reconstructed and generalized in the way just sketched, is right as far as the basic level of the world is concerned. If it is true that our basic physical theories give us knowledge only of the relations in which physical things stand, the metaphysics of intrinsic properties is in trouble: metaphysics has it that there are, at the basic level of the world, independent things, which are characterized by intrinsic properties each. On epistemological reflection, however, we have to concede that we do not have access to these properties insofar as they are intrinsic. A gap between metaphysics and epistemology thus arises.

If physics tells us only about the way in which the things at the basic level of the world are related to each other, two different metaphysical positions remain open:

1) One can continue to hold on to a metaphysics of intrinsic properties of the systems at the basic level of the world, but concede that we cannot gain any knowledge of these properties insofar as they are intrinsic.

2) One can give up a metaphysics of intrinsic properties in favour of a metaphysics of relations according to which the relations in which they stand are all there is to the things at the basic level.

The first one is the position that Jackson - somewhat reluctantly - endorses. As already mentioned in the introduction, the main argument for this position is that (a) relations require relata, that is, things which stand in the relations, and that (b) these things have to be something in themselves, that is, must have intrinsic properties over and above the relations in which they stand. Jackson makes use of this argument when he dismisses the view "that the nature of everything is relational cum causal, which makes a mystery of what it is that stands in the causal relations" (p. 24). However, as has been made clear in this paper, a metaphysics of relations has to reject merely the second part of this argument: one can maintain that (a) relations require relata, that is, things which stand in the relations, but that $\left(b^{*}\right)$ these things do not have any intrinsic properties over and above the relational properties, which can in principle be captured by physics. In this case, there is nothing paradoxical about a metaphysics of relations. In other words, there is no a priori argument that excludes a moderate - metaphysics of relations.

On the other hand, the adherent to a metaphysics of relations does not have any means at her disposal to rule out that there are some intrinsic properties or other of the related things. On an a priori basis, her claim can only be that, since her position is coherent, there is no argument left for maintaining that related things must of metaphysical necessity have some intrinsic property or other. Her a priori argument can only be that, applying Occam's razor, it is superfluous to include unknowable intrinsic properties in our account of the basic level of the world. However, in order to make a positive case for a metaphysics of relations, mere coherence is not enough. It is here that the metaphysical importance of the philosophy of quantum physics shows up: if we interpret quantum entanglement in terms of non-separability 
as sketched in this paper, we are entitled to maintain that the specific relations which our basic physical theory treats do not call for intrinsic properties of the related systems being admitted in our metaphysics, even if we cannot know these properties insofar as they are intrinsic. Thus, contrary to received opinion, quantum physics, in virtue of exhibiting entanglement, just provides us with the means to avoid a gap between epistemology and metaphysics: we can in principle know all there is at the basic level of the world; for what there is at the basic level of the world are relations of quantum entanglement.

\section{References}

Adams, R. M. (1979). Primitive thisness and primitive identity. Journal of Philosophy, 76, 5-26.

Black, M. (1952). The Identity of indiscernibles. Mind, 61, 153-164.

Cartwright, N. (1989). Nature's capacities and their measurement. Oxford: Oxford University Press.

Cleland, C. E. (1984. Space: An abstract system of non-supervenient relations. Philosophical Studies, 46, 19-40.

d'Espagnat, B. (1971). Conceptual foundations of quantum mechanics. Menlo Park: Benjamin.

Dipert, Randall R. (1997): “The mathematical structure of the world: The world as a graph". Journal of Philosophy 94, pp. 329-358.

Einstein, A., Podolsky, B. and Rosen, N. (1935). Can quantum-mechanical description of physical reality be considered complete? Physical Review, 47, 777-780.

Einstein, A. (1948). Quanten-Mechanik und Wirklichkeit. Dialectica, 2, 320-324.

Esfeld, M. (2001). Holism in philosophy of mind and philosophy of physics. Dordrecht: Kluwer.

French, S. (1989). Individuality, supervenience and Bell's theorem. Philosophical Studies, 55, 1-22.

French, S. and Ladyman, J. (2003). Remodelling structural realism: Quantum physics and the metaphysics of structure. Synthese, 136, 31-56.

French, S. and Redhead, M. L. G. (1988). Quantum physics and the identity of indiscernibles. British Journal for the Philosophy of Science, 39, 233-246.

Healey, R. A. (1991). Holism and nonseparability. Journal of Philosophy, 88, 393-421.

Howard, D. (1985). Einstein on locality and separability. Studies in History and Philosophy of Science, 16, 171201.

Howard, D. (1989). Holism, separability, and the metaphysical implications of the Bell experiments. In J. T. Cushing and E. McMullin (eds.), Philosophical consequences of quantum theory. Reflections on Bell's theorem (pp. 224-253). Notre Dame: University of Notre Dame Press.

Jackson, F. (1998). From metaphysics to ethics. A defence of conceptual analysis. Oxford: Oxford University Press.

Ladyman, J. (1998). What is structural realism? Studies in History and Philosophy of Modern Science, 29, 409424.

Langton, R. (1998). Kantian humility. Our ignorance of things in themselves. Oxford: Oxford University Press.

Langton, R. and Lewis, D. (1998). Defining 'intrinsic'. Philosophy and Phenomenological Research, 58, 333 345. Reprinted in D. Lewis (1999) Papers in metaphysics and epistemology. Cambridge: Cambridge University Press. Pp. 116-132.

Lewis, D. (1986a). Philosophical papers. Volume 2. Oxford: Oxford University Press.

Lewis, D. (1986b). On the plurality of worlds. Oxford: Blackwell.

Lewis, D. (2001). Redefining 'intrinsic'. Philosophy and Phenomenological Research, 63, 381-398.

Mermin, N. D. (1998). What is quantum mechanics trying to tell us? American Journal of Physics, 66, 753-767.

Müller, T. and Placek, T. (2001). Against a minimalist reading of Bell's theorem: Lessons from Fine. Synthese, $128,343-379$.

Rovelli, C. (1996). Relational quantum mechanics. International Journal of Theoretical Physics, 35, 1637-1678.

Teller, P. (1986). Relational holism and quantum mechanics. British Journal for the Philosophy of Science, 37, 71-81. 
Winsberg, E. and Fine, A. (2003). Quantum life: Interaction, entanglement, and separation. Journal of Philosophy, 100, 80-97.

Worrall, J. (1989). Structural realism: The best of two worlds? Dialectica, 43, 99-124. 